\title{
Uso del piso de rejilla como método físico de control de parásitos gastrointestinales en corderos recién destetados
}

\author{
Use of the grid floor as a physical method to control gastrointestinal \\ parasites in recently weaned lambs
}

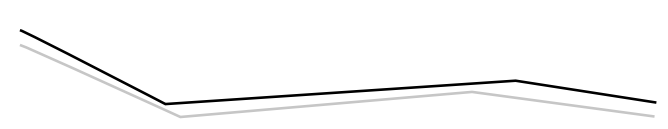

Francisco Escalera Valente*, Sergio Martínez González*, Fernando Benjamín Carrillo Díaz*, Socorro Marisa Salgado Moreno*, Fidel Ávila Ramos**, Agapito Gómez Gurrola*, José Lenin Loya Olguín*凶

Escalera Valente, F., Martínez González, S., Carrillo Díaz, F. B., Salgado Moreno, S. M. Ávila Ramos, F., Gómez Gurrola, A., \& Loya Olguín, J. L. (2017). Uso del piso de rejilla como método físico de control de parásitos gastrointestinales en corderos recién destetados. Investigación y Ciencia de la Universidad Autónoma de Aguascalientes, 25 (72), 12-19.

\section{RESUMEN}

El objetivo del presente trabajo fue evaluar el piso de rejilla para controlar parásitos gastrointestinales y determinar su efecto en algunas variables de producción. Se utilizaron 32 corderos divididos en dos grupos. El primero alojado en jaulas elevadas con piso de rejilla de plástico (PRP), el segundo en corrales con piso de concreto (PC). Semanalmente, se tomaron muestras de heces, se registró el consumo de alimento y la ganancia diaria de peso (GDP). A las heces se les realizó un análisis cualitativo y cuantitativo. No existió diferencia en el número de ooquistes/huevecillos de protozoarios (4,979 vs. $5,206)$, céstodos (125 vs. 56) ni de nemátodos (590 vs. 490) entre los grupos PRP y PC, respectivamente. Tampoco hubo diferencia en la GDP (240g PRP vs. $230 \mathrm{~g}$ PC) y en la conversión alimenticia (4.74 PRP vs.

Palabras clave: corderos; sanidad; resistencia parasitaria; piso de rejilla; producción.

Keywords: lambs; health; parasitic resistance; raisedslated floor; production.

\section{Recibido: 12 de enero de 2017, aceptado: 18 de agosto de 2017}

* Unidad Académica de Medicina Veterinaria y Zootecnia, Universidad Autónoma de Nayarit. Carretera de Cuota Compostela-Chapalilla km 3.5, C. P. 63700 , Compostela, Nayarit, México. Correo electrónico: franescalera@hotmail.com; sergiotepic@hotmail.com; fdoc_8@hotmail.com; coco_salgado@hotmail.com; yosibi@hotmail.com; joselenin28@hotmail. com

** Programa de Medicina Veterinaria y Zootecnia, División de Ciencias de la Vida, Universidad de Guanajuato campus Irapuato-Salamanca. Ex Hacienda El Copal km 9, C. P. 36500, Carretera Irapuato-Silao, A. P. 311 Irapuato, Guanajuato, México. Correo electrónico: ledifar@hotmail.com
4.75 PC). Bajo las condiciones del presente trabajo, no existe diferencia entre los corderos estabulados en piso de rejilla y en piso de concreto, en el control de parásitos gastrointestinales ni en el comportamiento productivo.

ABSTRACT

The objective was to evaluate the use of the raisedslated floor to control gastrointestinal parasites and to determine their effect on some production variables. Thirty two lambs divided into two groups were used. The first, housed in raised-slated floor (RSF), the second in pens with concrete floor (CF). Stool samples were taken weekly, food consumption and daily weight gain (DWG) were recorded. A qualitative and quantitative analysis were performed on the feces. There was no difference in the number of protozoan oocysts $(4,979$ vs. 5,206), cestodes (125 vs. 56) or nematodes (590 vs. 490) between the RSF and CF groups, respectively. There was also no difference on DGW (240g RSF vs. $230 \mathrm{~g} \mathrm{CF}$ ) and feed conversion (4.74 RSF vs. $4.75 \mathrm{CF}$ ). Under conditions of the present work there is no difference between lambs on grid floor and lambs on concrete floor in the gastrointestinal parasite control, neither in the productive behavior.

\section{INTRODUCCIÓN}

La producción de carne ovina en México no satisface la demanda del país, por tanto, se importa carne en canal y borrego en pie (Hernández Cortázar, Rejón Ávila, Valencia Heredia, \& Araujo Andrade, 


\section{IIVESTIGAGIÓn Y CUERCIA DE LA UกIVERSIDAD AUTÓOOOMA DE RGUASCALIERTES}

2014). El déficit de producción en México se debe principalmente al sistema de engorde de los corderos, la deficiencia de las instalaciones y la incidencia de enfermedades (Martínez González et al., 2011). El principal problema de salud al que se enfrentan los ovinos en México se debe a las enfermedades parasitarias (Mireles Martínez, Valencia Almazán, \& Gutiérrez Segura, 2009) que afectan con mayor frecuencia a los animales jóvenes y en desarrollo, provocan baja ganancia de peso y retraso en el crecimiento (González Garduño, Córdova Pérez, Torres Hernández, Mendoza de Gives, \& Arece García, 2011).

Dentro de las enfermedades causadas por parásitos, la coccidiosis es especialmente importante en corderos predestetados, recién destetados (Kaya, 2004) y en cría intensiva, ya que produce enfermedad entérica, pues dichos organismos invaden y destruyen las células epiteliales intestinales (Saratsis et al., 2013). Cuéllar-Ordaz (2008) reportó disminución de los problemas de salud por coccidiosis al usar corrales con piso de rejilla, un ejemplo de este uso se puede ver en la figura 1.

No obstante, en la mayoría de las investigaciones en corderos estabulados en piso de rejilla sólo se evalúa el comportamiento productivo (Lupton, Huston, Craddock, Pfeiffer, \& Polk, 2007; MagañaMonforte et al., 2015; Muñoz Osorio, Aguilar Caballero, Sarmiento Franco, Wurzinger, \& Gutiérrez Reynoso, 2015). Sin embargo, el comportamiento productivo de los animales no está totalmente desligado de las mermas que pueden ocasionar los parásitos (González Garduño et al., 2011 ).

Taylor (2013) comenta que debido al aumento de los reportes de resistencia a los antihelmínticos en todo el mundo, se requiere del desarrollo de estrategias de control parasitario que optimicen el uso de antihelmínticos. El uso del piso de rejilla puede ser una alternativa para disminuir la incidencia de las enfermedades parasitarias y contribuir a disminuir el uso de productos quimioterapéuticos, pues limita el contacto entre el hospedero y las formas infectantes de los parásitos; con ello, podrían mejorar las variables productivas de los corderos al destete. El objetivo de la investigación fue evaluar el uso del piso de rejilla para controlar parásitos gastrointestinales, mejorar la ganancia de peso y la conversión alimenticia en corderos recién destetados.

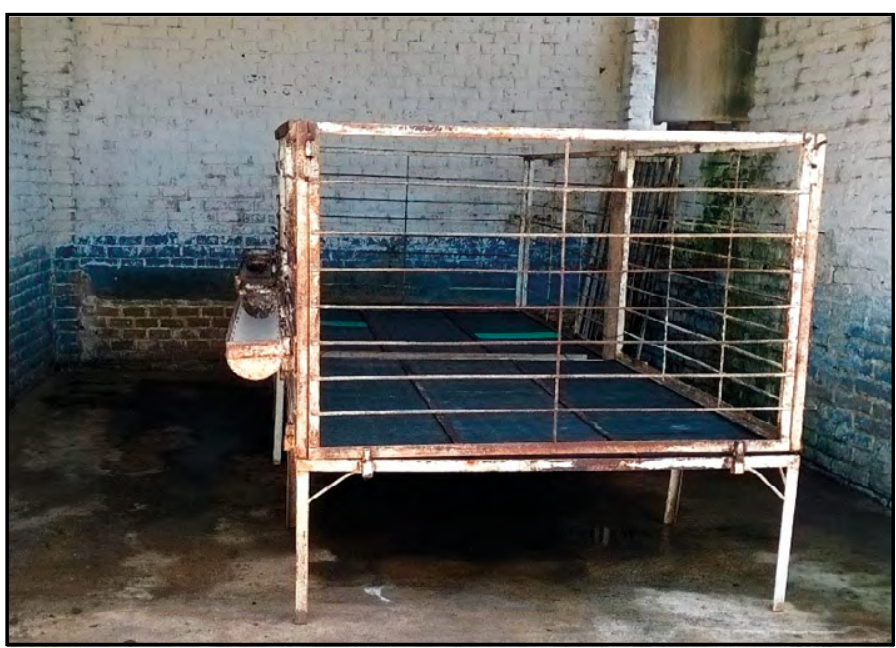

Figura 1. Jaula en elevado con piso de rejilla de plástico. Imagen del equipo de investigación.

\section{MATERIALES Y MÉTODOS}

\section{Ubicación del sitio experimental}

La investigación se realizó en la Unidad de Producción de Ovinos y Caprinos de la Unidad Académica de Medicina Veterinaria y Zootecnia de la Universidad Autónoma de Nayarit. Ubicada en Compostela, Nayarit $\left(21^{\circ} 14^{\prime} 14^{\prime \prime}\right.$ ' norte y $104^{\circ} 54^{\prime} 03^{\prime \prime}$ oeste) a 840 m.s.n.m., según el Instituto Nacional de Estadística y Geografía (INEGI, 2012). El manejo de los animales y la toma de las muestras se realizaron siguiendo los lineamientos de la Norma Oficial Mexicana NOM062-ZOO-1999 (2001); en cuanto a especificaciones técnicas para la producción, cuidado y uso de los animales de laboratorio, así como de explotaciones ganaderas, granjas, centros de producción, reproducción y cría.

\section{Unidades experimentales}

Se utilizaron 32 corderos (nacidos en la misma unidad de producción) de $73 \pm 6$ días de edad cruza Pelibuey x Katahdin: 16 hembras (12.2 $\pm 4.5 \mathrm{~kg})$ y 16 machos $(14.0 \pm 5.1 \mathrm{~kg})$. Las 32 unidades experimentales se pesaron al iniciar el experimento, se tomó una muestra de heces directamente del recto (utilizando guantes de látex esterilizados) y todos los corderos recibieron una dosis de selenio (Selen- $\mathrm{E}^{\oplus}, 1 \mathrm{ml} / 50 \mathrm{~kg}$ P.V. vía S.C.), como tratamiento preventivo de la enfermedad de músculo blanco. Se formaron dos grupos mixtos con ocho hembras y ocho machos; el primer grupo (11.88 $\pm 3.7 \mathrm{~kg})$ se colocó en jaulas con piso de rejilla (6.35 $\mathrm{m}^{2}$ cada jaula). El segundo se colocó ( $14.30 \pm 5.5 \mathrm{~kg}$ ) en corrales de piso de concreto (9.73 $\mathrm{m}^{2}$ cada corral). La etapa experimental duró 15 
IIVESTIGACIÓn Y CIERCIA DE LA UחIVERSIDAD AUTÓNOTH DE AGUASCALIERTES semanas dividida en dos periodos; el Periodo 1 fue durante las primeras siete semanas, el Periodo 2 duró ocho semanas.

Cada semana, a las 07:30 a.m. aproximadamente, todos los corderos fueron pesados antes de recibir el alimento, se les tomó una muestra de heces (10 g), se retiró y peso el alimento rechazado y por último se alimentaron con la dieta base a las 08:00 $h$, las dietas se elaboraron de acuerdo con las recomendaciones del National Research Council (NRC, 1985) para ovinos en etapa de destete. Las muestras de heces individuales se colocaron en bolsas estériles (Whirl-Pack Nasco) en condiciones de refrigeración para trasladarlas al laboratorio de Parasitología. La presencia y diferenciación de parásitos gastrointestinales se realizó mediante la técnica de flotación de Willis enriquecida, la cual permite maximizar la muestra en una laminilla hasta 100x; donde se distinguen estructuras como la forma, tamaño, número de blastómeros y la pared del huevo (Morales \& Pino, 2009). Para determinar el número de ooquistes por gramo de heces (opg) y huevecillos por gramo de heces (HPG) se utilizó la técnica de McMaster (Dryden, Payne, Ridley, \& Smith, 2005).

\section{Análisis estadístico}

Para analizar los datos se utilizó el diseño de bloques completos, bloqueado por sexo, con el modelo lineal general (GLM, por sus siglas en inglés) para ganancia de peso, el peso inicial de los corderos se usó como covariable. Además, se realizó un análisis de correlación entre las variables de comportamiento productivo y parasitológicas, se utilizó el paquete estadístico Statistical Package for the Social Sciences (SPSS) versión 20.0.

\section{RESULTADOS}

\section{Comportamiento parasitario}

Los géneros de los parásitos gastrointestinales (PGI) encontrados fueron protozoarios (Coccidia spp.), céstodos (Moniezia spp.) y nemátodos (Haemonchus contortus, Chabertia spp., Ostertagia spp., Trichostrongylus spp., Oesophagostomum spp., Paramphistomum spp., Toxocara spp., Cooperia spp.). Las cantidades totales de huevecillos/ooquistes de protozoarios, céstodos y nemátodos, fueron similares entre los dos grupos ( $p>0.05$ ) (tabla 1).

La interacción tipo de piso * sexo tampoco arrojó diferencias estadísticas ( $p>0.05$ ) en el número de huevecillos/ooquistes. Sin embargo, el número de nemátodos (sin importar el tipo de piso) fue mayor en machos que en hembras ( $p<0.05$ ), mientras que los protozooarios y los céstodos no mostraron diferencia $(p>0.05)$ (tabla 2$)$.

Tabla 1

Cantidad promedio (DS) de huevecillos/ooquistes encontrados en ovinos alojados en corrales de piso de rejilla o de concreto

\begin{tabular}{cccc}
\hline Tipo de piso & $\begin{array}{c}\text { Protozoarios } \\
\left(\text { opg }^{1}\right)\end{array}$ & $\begin{array}{c}\text { Céstodos } \\
\left(\mathrm{HPG}^{2}\right)\end{array}$ & $\begin{array}{c}\text { Nemátodos } \\
(\mathrm{HPG})\end{array}$ \\
\hline Piso de rejilla & $\begin{array}{c}4979 \\
( \pm 12739)\end{array}$ & $\begin{array}{c}125 \\
( \pm 1116)\end{array}$ & $\begin{array}{c}590 \\
( \pm 2502)\end{array}$ \\
$\begin{array}{c}\text { Piso de } \\
\text { concreto }\end{array}$ & $\begin{array}{c}5206 \\
( \pm 10920)\end{array}$ & $\begin{array}{c}56 \\
( \pm 240)\end{array}$ & $\begin{array}{c}490 \\
( \pm 2551)\end{array}$ \\
\hline
\end{tabular}

Nota: 'Ooquistes por gramo de heces. ${ }^{2}$ Huevecillos por gramo de heces. Elaboración propia.

Tabla 2

Número de huevecillos/ooquistes en hembras y machos de los diferentes grupos

\begin{tabular}{cccccccc}
\hline Grupo & $\begin{array}{c}\text { Piso de } \\
\text { rejilla }\end{array}$ & \multicolumn{2}{c}{$\begin{array}{c}\text { Piso de } \\
\text { concreto }\end{array}$} & \multicolumn{2}{c}{ Total } \\
\hline$M^{1}$ & $H^{2}$ & $M$ & $H$ & $M$ & $H$ \\
\hline $\begin{array}{c}\text { Protozoarios } \\
\text { (opg) }\end{array}$ & 4450 & 5384 & 4742 & 5792 & $4614^{a}$ & $5563^{a}$ \\
$\begin{array}{c}\text { Céstodos } \\
\text { (HPG) }\end{array}$ & 268 & 16 & 86 & 17 & $166^{a}$ & $17^{a}$ \\
$\begin{array}{c}\text { Nemátodos } \\
\text { (HPG) }\end{array}$ & 991 & 284 & 626 & 318 & $785^{a}$ & $299^{b}$ \\
\hline
\end{tabular}

Nota: 'Machos; ${ }^{2}$ Hembras. a, b Letras distintas en la misma fila indican diferencia significativa $(p<0.05)$.

Elaboración propia.

\section{Comportamiento productivo}

La tabla 3 presenta los resultados de las variables de peso inicial, peso final, ganancia total de peso, ganancia de peso diaria y conversión alimenticia. En ninguna variable productiva se observó diferencia ( $p>0.05$ ) entre los corderos en piso de concreto y piso de rejilla, aunque los corderos estabulados en piso de rejilla iniciaron con un peso $20 \%$ menor que los estabulados en piso de concreto.

\section{Correlaciones entre variables productivas y valores de HPG}

Con respecto a las correlaciones, ninguna de ellas 
Tabla 3

Comportamiento productivo de corderos Pelibuey x Katahdin recién destetados, alojados en corrales con piso de rejilla o concreto

\begin{tabular}{cccccc}
\hline Tipo de piso & PI & PF & GTP & GDP & CA (BS') \\
\hline Piso de rejilla & 11.88 & 36.2 & 23.80 & 0.240 & 4.74 \\
Piso de cemento & 14.30 & 36.9 & 22.00 & 0.230 & 4.75 \\
\hline
\end{tabular}

Nota: 'Base seca. $\mathrm{Pl}=$ peso inicial, $\mathrm{PF}=$ peso final, $\mathrm{GTP}=$ ganancia total de peso, $\mathrm{GDP}=$ ganancia diaria de peso, $\mathrm{CA}=$ conversión alimenticia.

Elaboración propia.

Tabla 4

Correlaciones presentadas entre las variables de

comportamiento productivo y huevecillos de parásitos

\begin{tabular}{|c|c|c|c|c|c|}
\hline & & $\mathrm{Pl}^{1}$ & $\mathrm{PF}^{2}$ & $\mathrm{GPT}^{3}$ & GDP $^{4}$ \\
\hline \multirow{3}{*}{$\begin{array}{c}\text { opg } \\
\text { protozoarios }\end{array}$} & $\begin{array}{l}\text { Correlación } \\
\text { de Pearson }\end{array}$ & -.216 & -.209 & .027 & -.237 \\
\hline & $\begin{array}{c}\text { Sig. } \\
\text { (bilateral) }\end{array}$ & .234 & .252 & .883 & .192 \\
\hline & $\mathrm{N}$ & 32 & 32 & 32 & 32 \\
\hline \multirow{3}{*}{$\begin{array}{l}\text { HPG } \\
\text { céstodos }\end{array}$} & $\begin{array}{l}\text { Correlación } \\
\text { de Pearson }\end{array}$ & -.125 & -.311 & -.202 & -.201 \\
\hline & $\begin{array}{c}\text { Sig. } \\
\text { (bilateral) }\end{array}$ & .494 & .083 & .268 & .269 \\
\hline & $\mathrm{N}$ & 32 & 32 & 32 & 32 \\
\hline \multirow{3}{*}{$\begin{array}{c}\text { HPG } \\
\text { nemátodos }\end{array}$} & $\begin{array}{l}\text { Correlación } \\
\text { de Pearson }\end{array}$ & .216 & .066 & -.230 & -.121 \\
\hline & $\begin{array}{c}\text { Sig. } \\
\text { (bilateral) }\end{array}$ & .236 & .722 & .205 & .510 \\
\hline & $\mathrm{N}$ & 32 & 32 & 32 & 32 \\
\hline
\end{tabular}

Nota: ${ }^{1} \mathrm{Pl}=$ Peso inicial. ${ }^{2} \mathrm{PF}=$ Peso final. ${ }^{3} \mathrm{GPT}=$ Ganancia de peso total. ${ }^{4}$ $\mathrm{GDP}=$ Ganancia diaria de peso.

Elaboración propia.

mostró diferencia estadística significativa ( $p>0.05)$, aunque la ganancia diaria de peso se correlaciona negativamente con el número de huevecillos/ooquistes de coccidia, céstodos y nemátodos (tabla 4).

\section{Análisis por periodos de estudio}

No existió diferencia significativa $(p>0.05)$ en el número de protozoarios entre los periodos de los dos grupos, aunque hubo tendencia a la disminución (figura 2).

Con respecto a los céstodos, no existió diferencia entre los periodos ( $p>0.05$ ), aunque se aprecia un incremento en el grupo de corderos colocados en piso de concreto y una disminución en los corderos colocados en piso de rejilla (figura 3).

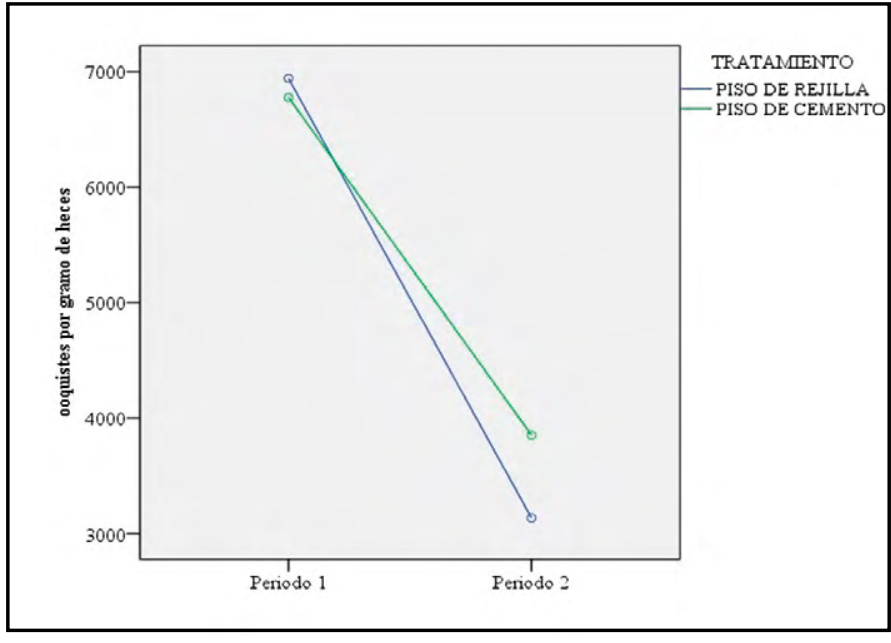

Figura 2. Número de ooquistes por gramo de heces de coccidia de corderos alojados en corrales con piso de rejilla o concreto.

Elaboración propia.

Por otro lado, los nemátodos de ambos tratamientos tampoco presentaron diferencia estadística $(p>0.05)$ entre los periodos. Sin embargo, es de notar que al inicio del experimento, los corderos del grupo estabulado en piso de rejilla presentaron un mayor número de huevecillos (figura 4).

\section{DISCUSIÓN}

\section{Comportamiento parasitario}

Los valores de coccidia fueron superiores a los de nemátodos y estos, a su vez, mayores a los céstodos ( $p<0.05)$, coincide con quienes describen a los protozoarios y nemátodos como las principales enfermedades parasitarias en corderos (Alzieu, Mage, Maes, \& De Muelenaere, 1999; González Garduño et al., 2011; Sayago et al., 2004). Los valores de coccidia encontrados por Sayago et al. (2004) previo al destete fueron muy elevados; sin embargo, en los muestreos posteriores al destete los valores disminuyeron a cerca de 1,000 opg, que son 
IIVESTIGACIÓn Y CIERGIA DE LA UNIVERSIDAD AUTÓNOMA

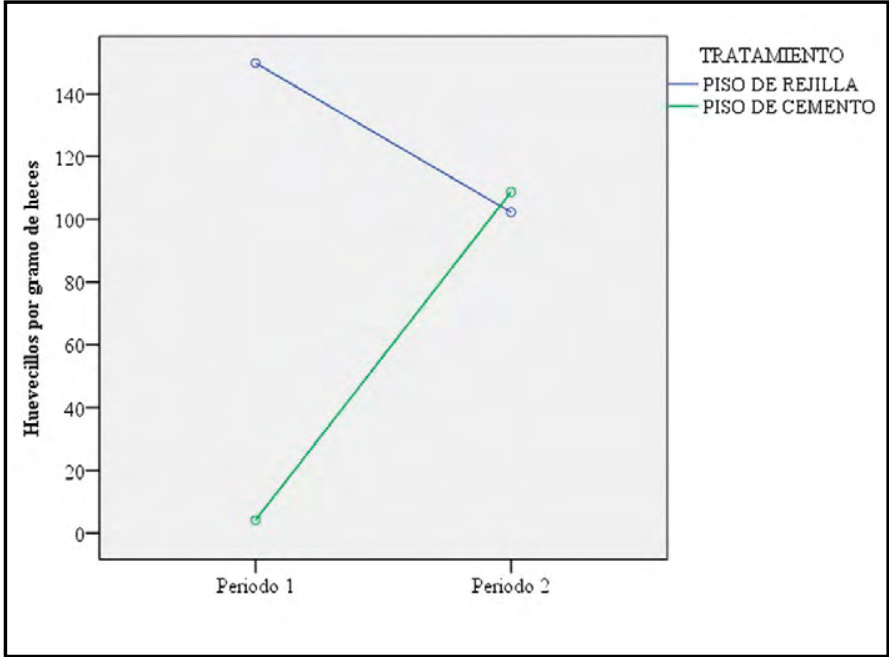

Figura 3. Número de huevecillos por gramo de heces de céstodos en corderos alojados en piso de rejilla o cemento.

Elaboración propia.

resultados inferiores a los valores de coccidia descritos en la presente investigación. No obstante (hay que recordar que estos autores dieron un tratamiento contra la coccidia), en este trabajo no se aplicó tratamiento coccicida. Por otro lado, los valores obtenidos son superiores a los publicados por Kaya (2004), quien describe un promedio de 3,540 ooquistes, esto pudo deberse al tipo de explotación utilizada, que pudo haber sido más higiénica; sin embargo, los autores no mencionan nada al respecto.

En cuanto a los céstodos, la mayoría de los trabajos realizados mencionan el porcentaje de prevalencia, pero no reportan el número de huevecillos encontrados por gramo de heces. En el estudio realizado por García Zambrano (2011) se reportan cargas parasitarias de Moniezia con valores previos al tratamiento de 505, 510 y $510 \mathrm{HPG}$ para el grupo testigo, tratado con albendazol y tratado con prazicuantel, respectivamente; mayores a las encontradas en la presente investigación en ambos grupos. El tipo de animales que utilizaron probablemente fueron hembras en pastoreo, lo que pudo determinar los valores más elevados. Se ha reportado que la reducción en la ganancia diaria de peso debida al parasitismo con Moniezia spp. puede ser de alrededor de $89 \mathrm{~g}$ por día (Mireles Martínez et al., 2009).

En el trabajo realizado por González Garduño et al. (2011) el conteo total de nemátodos gastrointestinales adultos fue de 2,175; en muestras

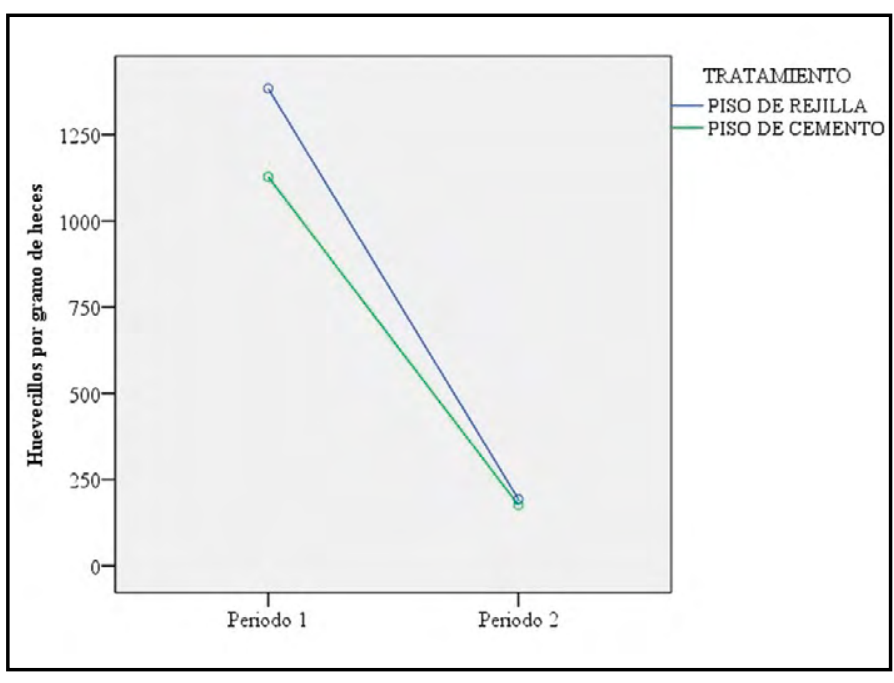

Figura 4. Número de huevecillos por gramo de heces de nemátodos en corderos alojados en piso de rejilla o cemento. Elaboración propia.

obtenidas, después del sacrificio de los animales, directamente de abomaso, intestino delgado e intestino grueso; estos valores son superiores a los encontrados en la presente.

La falta de diferencia estadística entre el grupo de rejilla y el de concreto, en cuanto al número de huevecillos/ooquistes de protozoarios, céstodos y nemátodos, pudo deberse al hecho de que los corrales de piso de cemento recibían una limpieza diaria, lo que disminuía la exposición de los corderos a las formas infectantes de los parásitos.

Con relación al de sexo de los corderos, no se obtuvo diferencia estadística $(p>0.05)$ en la interacción tipo de piso * sexo. Sin embargo, independientemente del tipo de piso, los machos presentaron mayores niveles de nemátodos ( $p<0.05$ ) que las hembras. Este hecho coincide con lo descrito por Idris, Moors, Sohnrey y Gauly (2012), quienes describen a los machos como más sensibles que las hembras. López Ruvalcaba, González Garduño, Osorio Arce, Aranda lbañez y Díaz Rivera (2013) encontraron que los machos de desecho fueron más parasitados por nemátodos que las hembras. La influencia del sexo en la susceptibilidad a las infecciones podría atribuirse a la predisposición genética y a la susceptibilidad diferencial debidas al control hormonal (Tariq, Chishti, Ahmad, \& Shawl, 2008). Estudios indican que la testosterona tiene un efecto inmunosupresor (Decristophoris, Hardenberg, \& McElligot, 2007; Muehlenbein \& Watts, 2010). 


\section{IIVESTIGAGIÓn Y CUERCIA DE LA UחIVERSIDAD AUTÓNOMA DE AGUASCALIERTES}

\section{Comportamiento productivo}

En comparación con los trabajos realizados en jaulas con piso de rejilla, la GDP observada es menor que las de Muñoz Osorio et al. (2015) y Garza Cedillo, Garza Caballero y Hernández Rojas (2013), de $263 \mathrm{~g}$ y $271 \mathrm{~g}$, respectivamente; pero similar a la encontrada por Magaña-Monforte et al. (2015) (229 g/día en animales Katahdin $x$ Pelibuey) y superior a la descrita por Lupton et al. (2007) (160 g). En su estudio, Muñoz Osorio et al. (2015) utilizaron un desparasitante en los corderos antes de iniciar el trabajo, lo que pudo determinar su mejor GDP. Magaña-Monforte et al. (2015) también desparasitaron los animales antes de empezar; sin embargo, utilizaron un porcentaje de proteína más bajo que el del presente (16\% vs. $17.9 \%$ de PC, respectivamente), lo que pudo resultar en una GDP similar.

La mejor ganancia diaria de peso (118.76 g) encontrada por Sayago et al. (2004) fue en el grupo tratado con sulfas, mismo que promedió 10,342 ooquistes de coccidia. La GDP que se obtuvo fue prácticamente el doble, puntualizando que el número de ooquistes fue prácticamente la mitad. También se han realizado trabajos con extractos naturales y su efecto contra las poblaciones de Eimeria spp., como el realizado por CervantesValencia, Alcalá-Canto, Sumano-Lopez, DucoingWatt y Gutiérrez-Olvera (2016) en el que probaron el extracto de Curcuma longa, encontraron que la dosis más alta $(200 \mathrm{mg} / \mathrm{kg})$ favoreció la reducción de coccidias y logró la mayor ganancia diaria de peso (185 g) con respecto al resto de tratamientos; sin embargo, dicha ganancia es inferior a la encontrada en el presente trabajo.

A pesar de que el espacio de las jaulas con piso de rejilla era menor $\left(0.79 \mathrm{~m}^{2}\right.$ por cordero) que en las de piso de cemento ( $1.21 \mathrm{~m}^{2}$ por cordero) y de que al inicio del trabajo el grupo testigo pesaba $2.42 \mathrm{~kg}$ más que el grupo tratado (diferencia que se vio reducida al final de la engorda, siendo apenas de $0.7 \mathrm{~kg}$ ), el promedio en la ganancia de peso total fue $7.56 \%$ mayor en los animales estabulados en piso de rejilla
(23.8 kg) que en los alimentados en piso de concreto $(22.0 \mathrm{~kg})$. Otro aspecto es la optimización de la mano de obra, pues mientras que en los corrales de piso de concreto se requería de limpieza diaria, en el piso de rejilla se puede realizar en menos ocasiones y más rápido.

\section{Correlaciones entre variables productivas y valores de HPG}

Las correlaciones entre la ganancia diaria de peso y el número de coccidia fueron negativas (aunque no significativas). Los resultados concuerdan con quienes establecen que la presencia de parásitos disminuye la ganancia de peso (Mireles Martínez et al., 2009). Gauly, Reeg, Baver y Erhardt (2004) determinaron una correlación negativa entre el número de ooquistes de Eimeria y la ganancia diaria de peso. En el trabajo de Reeg et al. (2005) no estudiaron la ganancia diaria de peso; sin embargo, sí determinaron una correlación negativa entre el peso de los corderos, en las diferentes etapas, y los valores de ooquistes por gramo de heces de las diferentes especies de Eimeria.

\section{Análisis por periodos de estudio}

La falta de diferencias estadísticas, en cuanto a los nemátodos entre ambos periodos, coincide con Idris et al. (2012) y con Esteban-Andrés et al. (2013), pues en el primer grupo se encontró una correlación negativa entre la edad de los corderos y el número de huevecillos, y en el segundo una disminución de los conteos de nemátodos conforme transcurría el tiempo. Kambara y McFarlane (1996) mencionan que existe mejora en el desarrollo de la inmunidad a mayor edad.

CONCLUSIONES

Bajo las condiciones del presente trabajo no existe diferencia entre los corderos estabulados en piso de rejilla y en piso de concreto en el control de parásitos gastrointestinales ni en el comportamiento productivo. 
- Alzieu, J. P., Mage, C., Maes, L., \& De Mûelenaere, C. (1999). Economic benefits of prophylaxis with diclazuril against subclinical coccidiosis in lambs reared indoors. Veterinary Record, 144, 442-444. doi: 10.1136/vr.144.16.442

- Cervantes-Valencia, M., Alcalá-Canto, Y., Sumano-Lopez, H., Ducoing-Watty, A., \& Gutierrez-Olvera, L. (2016). Effects of Curcuma longa dietary inclusion against Eimeria spp. in naturally-infected lambs. Small Ruminant Research, 136, 27-35. doi: 10.1016/j.smallrumres.2015.12.035

- Cuéllar-Ordaz, J. A. (2008). Uso de pisos de rejilla en explotaciones ovinas [Ficha tecnológica]. En Fortalecimiento del Sistema Producto Ovinos. Tecnologías para ovinocultores (pp 146-148). México: Asociación Mexicana de Criadores de Ovinos. Recuperada el 19 de agosto de 2017, de http://www. uno.org.mx/sistema/pdf/produccion/usodepisosderejilla.pdf

- Decristophoris, P. M. A., von Hardenberg., A., \& McElligott, A. G. (2007). Testosterone is positively related to the output of nematode eggs in male alpine ibex (Capra ibex) faeces. Evolutionary Ecology Research, 9, 1277-1292. doi: 10.5167/uzh714

- Dryden, M. W., Payne, P. A., Ridley, R., \& Smith, V. (2005). Comparison of common fecal flotation techniques for the recovery of parasite eggs and oocysts. Veterinary Therapeutics: Research in Applied Veterinary Medicine, 6(1), 15-28.

- Esteban-Andrés, D., González-Garduño, R., Garduza-Arias, G., Ojeda-Robertos, N., Reyes-Montes, F., \& Gutiérrez-Cruz, S. (2013). Desarrollo de resistencia a nematodos gastrointestinales en ovinos de pelo desafiados con diferentes niveles de infección. Revista de la Facultad de Medicina Veterinaria y de Zootecnia, 60(3), 169-181.

- García Zambrano, S. P. (2011). Estudio sanitario-productivo de la afección endoparasitaria por cestodos en ovinos mestizos. Tesis de Grado. Riobamba, Ecuador: Escuela Superior Politécnica de Chimborazo.

- Garza Cedillo, R., Garza Caballero, M., \& Hernández Rojas, P. (octubre, 2013). Evaluation of two systems of broiler intensive hair sheep in northern Tamaulipas. XVII Congreso Internacional de Ovinocultura, 23-25 de octubre, Acapulco, Guerrero, 126-130.

- Gauly, M., Reeg, J., Baver, C., \& Erhardt, G. (2004). Influence of production systems in lambs on the Eimeria oocyst output and weight gain. Small Ruminant Research, 55(13), 159-167. Recuperado de http://dx.doi.org/10.1016/j. smallrumres.2004.02.001

- González Garduño, R., Córdova Pérez, C., Torres Hernández, G., Mendoza de Gives, P., \& Arece García, J. (2011). Prevalencia de parásitos gastrointestinales en ovinos sacrificados en un rastro de Tabasco, México. Veterinaria México, 42(2), 125-135.

- Hernández Cortázar, I., Rejón Ávila, M., Valencia Heredia, E., \& Araujo Andrade, L. (2014). Análisis de inversión para la producción de ovinos en el municipio de Tzucacab, Yucatán, México. Revista Mexicana de Agronegocios, 18(34), 677-687.

- Idris, A., Moors, E., Sohnrey, B., \& Gauly, M. (2012). Gastrointestinal nematode infections in German sheep. Parasitology Research, 110(4), 1453-1459. Recuperado de http://dx.doi.org/10.1007/ s00436-01 1-2648-1

- Instituto Nacional de Estadística y Geografía. (2012). Anuario estadístico del estado de Nayarit. México: Gobierno del estado de Nayarit.

- Kambara, T., \& McFarlane, R. G. (1996). Changes in T cell subpopulations of sheep due to age and dietary protein intake; association with protective immunity to Trichostrongylus colubriformis. Veterinary Immunology and Immunopathology, 51 (1-2), 127-135. doi: 10.1016/0165-2427(95)05513-4

- Kaya, G. (2004). Prevalence of Eimeria species in lambs in Antakya Province. Turkish Journal of Veterinary \& Animal Sciences, 28(4), 687-692.

- López Ruvalcaba, O. A., González Garduño, R., Osorio Arce, M. M., Aranda Ibañez, E., \& Díaz Rivera, P. (2013). Cargas y especies prevalentes de nematodos gastrointestinales en ovinos de pelo destinados al abasto. Revista Mexicana de Ciencias Pecuarias, 4(2), 223-234.

- Lupton, C. J., Huston, J. E., Craddock, B. F., Pfeiffer, F. A., \& Polk, W. L. (2007). Comparison of three systems for concurrent production of lamb meat and wool. Small Ruminant Research, 72(2-3), 133-140. doi: 10.1016/j.smallrumres.2006.10.002

- Magaña-Monforte, J. G., Moo-Catzin, C. J., Chay-Canul, A. J., Aké-López, J. R., Segura-Correa, J. C., \& Montés-Pérez, R. C. (2015). Crecimiento y componentes de la canal de ovinos de pelo en jaulas elevadas. Livestock Research for Rural Development, 27(6). Recuperado de http://www.Irrd.org/ Irrd27/6/maga27104.html

- Martínez González, S., Macías Coronel, H., Moreno Flores, L. A., Zepeda García, J., Espinoza Moreno, M. E., Figueroa Morales, R., \& Ruiz Félix, M. (2011). Análisis económico en la producción de ovinos en Nayarit, México. Abanico Veterinario, 1 (1), 37-43.

- Mireles Martínez, E. J., Valencia Almazán, M. T., \& Gutiérrez Segura, I. (2009). Parasitosis gastrointestinal natural y la ganancia diaria de peso de corderos lactantes en el trópico seco de Guerrero, México. Revista Electrónica de Veterinaria, 11 (1), 1-9. Recuperado de http://www.veterinaria.org/revistas/ redvet/n010110/011002.pdf 


\section{IIVESTIGAGIÓn Y CUERGIA DE LA UחIVERSIDAD AUTÓNOMTH DE AGUASCALIERTES}

- Morales, G., \& Pino, L. A. (2009). Nematodes parásitos de los rumiantes domésticos en Venezuela. Diagnóstico y control (143 pp.). Caracas, Venezuela: Laboratorio de Diagnóstico Veterinario "ALIANI".

- Muehlenbein, M. P., \& Watts, D. P. (2010). The costs of dominance: testosterone, cortisol and intestinal parasites in wild male chimpanzees. BioPsychoSocial Medicine, 4(1), 21. doi: 10.1186/1751-0759-4-21

- Muñoz-Osorio, G. A., Aguilar-Caballero, A. J., SarmientoFranco, L. A., Wurzinger, M., \& Gutiérrez-Reynoso, G. A. (2015). Factores que influyen sobre algunas variables productivas en corderos finalizados en corrales elevados con piso de rejilla. Nova Scientia, 7(15), 285-296. doi: 10.21640/ns.v7i15.342

- National Research Council. (1985). Nutrient requirements of sheep (6a. ed.). Washington, DC: National Academy Press.

- Norma Oficial Mexicana NOM-062-ZOO-1999. Especificaciones técnicas para la producción, cuidado y uso de los animales de laboratorio. Diario Oficial de la Federación, Distrito Federal, México, 22 de agosto de 2001.

- Reeg, K. J., Gauly, M., Baver, C., Mertens, C., Erhardt, G., \& Zahner, H. (2005). Coccidial infections in housed lambs: oocyst excretion, antibody levels and genetic influences on the infection. Veterinary Parasitology, 127(3-4), 209-219. doi: 10.1016/j.vetpar.2004.10.018

- Saratsis, A., Karagiannis, I., Brozos, C., Kiossis, E., Tzanidakis, N., Joachim, A., \& Sotiraki, S. (2013). Lamb eimeriosis: Applied treatment protocols in dairy sheep production systems. Veterinary Parasitology, 196(1-2), 56-63. doi: 10.1016/j. vetpar.2013.01.020

- Sayago, D., Ríos de Álvarez, L., Rondón, Z., García, F., Colmenares, O. A., Machado, I., \& Muñoz, G. (2004). Uso de controles profilácticos y terapéuticos de coccidios en corderos tropicales. Revista de la Facultad de Ciencias VeterinariasUniversidad Central de Venezuela, 45(1), 45-54.

- Tariq, K. A., Chishti, M. Z., Ahmad, F., \& Shawl, A. S. (2008). Epidemiology of gastrointestinal nematodes of sheep managed under traditional husbandry system in Kashmir valley. Veterinary Parasitology, 158(1-2), 138-143. doi: 10.1016/j. vetpar.2008.06.013

- Taylor, M. A. (2013). Parasite control in sheep: A risky business. Small Ruminant Research, 110(2-3), 88-92. doi: 10.1016/j. smallrumres.2012.11.010 